\title{
Modeling of Picosecond Laser-induced Plasma Amplification inside a Microhole and an Implied Novel Technology to Drill Microholes with Varying Diameters with Depth
}

\author{
${ }^{\mathrm{a}}$ Navid Dabir-Moghaddam, ${ }^{\mathrm{b}}$ Sha Tao, *Benxin Wu, and ${ }^{\mathrm{c}}$ Yung C. Shin \\ ${ }^{\text {a }}$ Illinois Institute of Technology, Chicago IL \\ ${ }^{\mathrm{b}}$ Advanced Optowave Corp., Ronkonkoma NY \\ ${ }^{\mathrm{c}}$ Purdue University, West Lafayette IN \\ *Corresponding author; Adjunct Associate Professor, Illinois Institute of Technology, Chicago \\ IL, email: bwu11@iit.edu; Associate Professor, School of Mechanical Engineering, Purdue \\ University, 585 Purdue Mall, West Lafayette, IN 47907, email: wu65@purdue.edu
}

\begin{abstract}
Microholes with varying diameters at different depths are very desirable in various important applications. However, it is very challenging to produce microholes with varying diameters when the variation is in a complicated way and/or when the hole diameter is very small. This paper presents physics-based modeling work on the interactions among a picosecond (ps) laser pulse, a pre-existing plasma plume inside a microhole, and the hole sidewall. The modeling work implies the potential feasibility of a novel dual-pulse laser ablation and plasma amplification (LAPA) process for drilling microholes with varying diameters at different depths.
\end{abstract}

Keywords: laser drilling; laser micromachining

\section{Introduction}

Many important industrial applications may need holes with microscale diameters, and some specific examples include the small orifices needed in fuel injectors for diesel engines [1] and the holes for the cooling purpose in aerospace engines [2, 3]. Microholes with diameters that vary with the hole depth in certain ways may be very desirable in some applications. For example, microholes with small diameters that vary with the hole depth (such as a reverse tapered hole where the hole diameter increases with the hole depth, or a dumbbell-shaped hole where the hole diameter first decreases and then increases with the hole depth), if applied in diesel fuel injectors, may enhance the fuel atomization and result in a more complete combustion [1]. However, drilling microholes that have diameters varying with the hole depth is very challenging when the diameter variation with the depth is complicated (such as a dumbbellshaped hole) and/or when the hole diameter is very small. 
To study the potential feasibility of a new technology for drilling microholes that have small diameters varying with the hole depth, in this paper modeling work has been performed on the interactions among a picosecond (ps) laser pulse, a pre-existing plasma plume inside a microhole, and the microhole sidewall. In the model, two-dimensional (2D) axi-symmetric gas dynamic equations are solved for the gaseous phase inside the microhole (including the plasma and the ambient gas), while a 2D axi-symmetric heat transfer equation is solved for the hole sidewall region. The gas dynamic equations and the heat transfer equation are coupled through the heat flux from the gaseous phase to the sidewall surface. It has been found that the ps laser pulse can amplify the pre-existing plasma (that is, increase the plasma temperature), and hence increase the heat flux from the gaseous phase to the sidewall surface, which may cause spatially selective material removal from the sidewall. This implies the potential feasibility of drilling microholes with varying diameters at different depths through a novel technology, called dualpulse laser ablation and plasma amplification (LAPA), which will be introduced later in this paper.

\section{Model}

Figure 1 shows the setup of the model. It is assumed that at $t=0$ an aluminum plasma plume pre-exists inside a microhole whose side and bottom walls are made of solid aluminum. The hole has a diameter of $50 \mu \mathrm{m}$, and the hole bottom is located at $\mathrm{z}=0$, and the hole sidewall surface is located at $\mathrm{r}=25 \mu \mathrm{m}$. The pre-existing plasma has an initial height of $150 \mu \mathrm{m}$, an initial diameter of $40 \mu \mathrm{m}$, and an initial temperature of $100000 \mathrm{~K}$ (which is close to the earlystage temperature reported in [4] for plasma induced by nanosecond laser ablation of aluminum). The plasma region is surrounded by ambient argon gas at an initial temperature of $300 \mathrm{~K}$ and an initial pressure of 1 atmosphere. At $\mathrm{t}=0$, it is assumed that a 1064-nm laser beam with a 100-ps 
full-width-at-half-maximum (FWHM) pulse duration propagates downwards and starts interacting with the plasma. The laser beam intensity has a top-flat spatial distribution and the beam diameter is $40 \mu \mathrm{m}$. The model calculation starts at $\mathrm{t}=0$. The evolution of the gaseous phase inside the microhole (including the aluminum plasma and the ambient argon) is simulated by solving the $2 \mathrm{D}$ axi-symmetric gas dynamic equations [5-9]:

$\frac{\partial \rho_{1}}{\partial t}+\frac{1}{r} \frac{\partial\left(r \rho_{1} v_{r}\right)}{\partial r}+\frac{\partial\left(\rho_{1} v_{z}\right)}{\partial z}=0$

$\frac{\partial \rho_{2}}{\partial t}+\frac{1}{r} \frac{\partial\left(r \rho_{2} v_{r}\right)}{\partial r}+\frac{\partial\left(\rho_{2} v_{z}\right)}{\partial z}=0$

$\frac{\partial\left(\left(\rho_{1}+\rho_{2}\right) v_{r}\right)}{\partial t}+\frac{1}{r} \frac{\partial\left(r\left(\rho_{1}+\rho_{2}\right) v_{r} v_{r}\right)}{\partial r}+\frac{\partial\left(\left(\rho_{1}+\rho_{2}\right) v_{r} v_{z}\right)}{\partial z}=-\frac{\partial P}{\partial r}$

$\frac{\partial\left(\left(\rho_{1}+\rho_{2}\right) v_{z}\right)}{\partial t}+\frac{1}{r} \frac{\partial\left(r\left(\rho_{1}+\rho_{2}\right) v_{r} v_{z}\right)}{\partial r}+\frac{\partial\left(\left(\rho_{1}+\rho_{2}\right) v_{z} v_{z}\right)}{\partial z}=-\frac{\partial P}{\partial z}$

$\frac{\partial\left(E_{k}+E_{i n}\right)}{\partial t}+\frac{1}{r} \frac{\partial\left(r\left(E_{k}+E_{i n}+P\right) v_{r}\right)}{\partial r}+\frac{\partial\left(\left(E_{k}+E_{i n}+P\right) v_{z}\right)}{\partial z}=\nabla \cdot\left(k_{e} \nabla T\right)+\alpha_{I B} I$

where $t$ denotes time, $\mathrm{r}$ and $\mathrm{z}$ are spatial coordinates, $\rho_{1}$ and $\rho_{2}$ represent the density of aluminum plasma and argon, respectively, $\mathrm{P}$ represents pressure, $v_{r}$ and $v_{z}$ are the velocity components in $\mathrm{r}$ and $\mathrm{z}$ directions, respectively, $E_{k}$ and $E_{i n}$ denote the kinetic energy and the internal energy, respectively, $T$ is temperature, $k_{e}$ represents electron thermal conductivity [10-11] (for ambient argon at temperatures close to its initial temperature of $300 \mathrm{~K}$, the argon conductivity at $300 \mathrm{~K}$ is applied), $\alpha_{I B}$ is the inverse bremsstrahlung optical absorption coefficient [10-12], and $I$ denotes the laser beam intensity.

To solve the gas dynamics equations, related equation of state (EOS) data is needed, which is developed based on the electron number density obtained by solving the Saha equation [13-14]. 
Due to the heat transfer between the gaseous phase in the microhole and the hole sidewall surface, the sidewall temperature may change. The spatial distribution and temporal evolution of the temperature in the sidewall is obtained by solving the $2 \mathrm{D}$ axi-symmetric heat transfer equation in the sidewall $[5,9,15-18]$. When the sidewall surface is melted and the surface temperature is sufficiently high, surface vaporization may become significant. The surface vaporization flux can be calculated using the Hertz-Knudsen equation [18-19] based on the sidewall surface temperature. The surface vaporization flux, divided by the density of the vaporizing sidewall surface and integrated with time, can yield the accumulated vaporization depth.

The gas dynamic equations are solved numerically using a finite difference essentially non-oscillatory (ENO) scheme [20], and the heat transfer equation in the sidewall is solved using the explicit finite volume method $[7,16]$. At each numerical time step, by solving the gas dynamic equations, the gaseous phase velocity, density, and temperature distributions can be obtained. Then the heat conduction flux from the gaseous phase to the microhole sidewall surface can be determined, based on which the heat transfer equation in the sidewall is solved to get the temperature distribution of the sidewall at this time step. Then, the numerical solution for the next time step will be performed. In solving all the equations, the needed material property information, etc., is taken from the literature [16, 18, 21-23].

\section{Results and Discussions}

Due to the heat transfer from the gaseous phase in the microhole to the hole sidewall surface, the sidewall surface melting and vaporization may occur. In the $2 \mathrm{D}$ axi-symmetric 
coordinate system (as shown in Fig.1), each sidewall surface point corresponds to a certain $\mathrm{z}$ coordinate, and also has a corresponding accumulated vaporization depth. The accumulated vaporization depth (by $\mathrm{t}=3 \mathrm{~ns}$ ) versus $\mathrm{z}$ is plotted in Fig. 2 (by $\mathrm{t}=3 \mathrm{~ns}$, the sidewall surface vaporization velocity has decreased to a very small value and further increase of the accumulated vaporization depth with time, if any, is extremely slow). It has been found in the model calculations that if no picosecond (ps) laser pulse is applied, then the accumulated vaporization depth is negligibly small and is not shown in the figure. However, when a ps laser pulse is applied at $\mathrm{t}=0$, the laser pulse will amplify the plasma (that is, increase the plasma temperature), and hence increase the heat flux to the sidewall. As a result, obvious vaporization may occur. Figure 2 shows that when the ps laser pulse fluence is $6 \mathrm{~J} / \mathrm{cm}^{2}$, the sidewall surface vaporization mainly occurs in the region of $\mathrm{z}=\sim 100$ to $150 \mu \mathrm{m}$. The peak vaporization depth occurs at $\mathrm{z}=$ $\sim 128 \mu \mathrm{m}$, which is slightly below the initial plasma top $\mathrm{z}$ coordinate of $150 \mu \mathrm{m}$. Vaporization has also occurred below $\mathrm{z}=100 \mu \mathrm{m}$, but the accumulated vaporization depth is relatively very small. When the ps laser pulse has a higher fluence of $8 \mathrm{~J} / \mathrm{cm}^{2}$, the peak vaporization depth is much larger than that for $6 \mathrm{~J} / \mathrm{cm}^{2}$, but still occurs at a similar location of $\mathrm{z}=\sim 128 \mu \mathrm{m}$. Most of the vaporization still occurs in the region of $\mathrm{z}=\sim 100$ to $150 \mu \mathrm{m}$, but the vaporization in the region of $\mathrm{z}=\sim 50$ to $100 \mu \mathrm{m}$ also becomes obvious.

Figure 3 shows the temporal evolution of the heat flux to the sidewall surface at the peak vaporization depth location of $\mathrm{z}=128 \mu \mathrm{m}$. It can be seen that when no ps laser pulse is applied, the peak heat flux is only around $\sim 1.4 \times 10^{12} \mathrm{~W} / \mathrm{m}^{2}$. However, when a ps laser pulse is applied, the peak flux increases to $\sim 5.5 \times 10^{12} \mathrm{~W} / \mathrm{m}^{2}$ (for $6 \mathrm{~J} / \mathrm{cm}^{2}$ laser pulse fluence) and to $\sim 6.7 \times 10^{12}$ $\mathrm{W} / \mathrm{m}^{2}$ (for $8 \mathrm{~J} / \mathrm{cm}^{2}$ laser pulse fluence). The heat flux first increases to a peak value and then decreases with time. The heat flux FWHM pulse duration is around $\sim 0.5 \mathrm{~ns}$. The heat flux shown 
in Fig. 3 has helped the understanding of the different peak vaporization depths for different ps laser fluences shown in Fig. 2, and also the understanding of why no significant vaporization occurs when no ps laser pulse is applied.

Figure 4 shows the spatial distribution of heat flux to the sidewall surface at $t=0.44 \mathrm{~ns}$ (which is the time when peak heat flux occurs for $8 \mathrm{~J} / \mathrm{cm}^{2}$ in Fig.3). When no ps laser pulse is applied, the heat flux is relatively uniform spatially for most of the regions below $\mathrm{z}=\sim 150 \mu \mathrm{m}$. When a ps laser pulse is applied, due to the absorption of the laser pulse energy by the plasma, the plasma temperature has been increased and hence the heat flux to the sidewall surface has been increased significantly. Figure 4 shows that a higher ps laser pulse fluence leads to a higher heat flux to the sidewall surface. The application of a ps laser pulse has also made the spatial distribution of the heat flux very non-uniform. The heat flux has a peak value in the region slightly below $\mathrm{z}=\sim 150 \mu \mathrm{m}$, and then the heat flux decreases as the $\mathrm{z}$ coordinate decreases. The spatial non-uniformity of the heat flux has helped the understanding of the spatial variation of the vaporization depths shown in Fig. 2.

In summary, under the studied conditions the model calculations show that the ps laser pulse can amplify the pre-existing plasma (that is, increase the plasma temperature), and spatially non-uniformly increase the heat flux to the hole sidewall surface, which can induce spatially selective material removal from the hole sidewall. This may make the hole diameters different at different depths. The simulation results imply the potential feasibility of a novel technology to drill microholes with varying diameters at different depths, that is, the so-called dual pulse laser ablation and plasma amplification (LAPA) process. In the dual pulse LAPA process, (i) a short laser pulse is first sent to ablate the bottom of a microhole to increase the depth of the hole and produce a plasma plume, and then (ii) a second short laser pulse is sent to amplify the plasma to 
selectively remove materials from the hole sidewall to change the hole diameter. By repeating (i) an (ii) with suitable and variable (if needed) parameters of the two laser pulses (including the timing of the second laser pulse and even the possible application of additional plasmaamplifying laser pulses besides the second pulse, if needed), potentially a microhole with varying diameters at different depths can be produced. Even a complicated diameter variation with the depth may be potentially produced, such as a dumbbell-shaped hole. Laser beam can be focused to a very small spot and hence LAPA may have a high spatial resolution, and may potentially produce microholes very small diameters varying with the depth. LAPA may be applied to both electrically conductive and non-conductive materials, and does not have mechanical tool wear problems. Certainly, although the theoretical modeling work in this paper implies the potential feasibility of LAPA, experimental work and further modeling work are still needed to completely confirm LAPA's feasibility, fully understand its underlying physical mechanisms, and reveal its major actual advantages and disadvantages.

It should be noted that the major goal of this paper is to perform modeling work to study the potential feasibility of LAPA, and it is not the goal of this paper to develop a quantitatively completely accurate model. The model in this paper is based on several simplified assumptions, and it is expected that the assumptions will not significantly affect the accomplishment of the major goal of this paper mentioned above (certainly, future work may still be needed to completely confirm this). For example, in the model the possible laser beam reflection at the plasma top front is neglected, and hence each laser pulse fluence given in this paper is the fluence of the incoming laser beam minus the reflected beam (if any) at the plasma top front. When laser beam propagates to the hole bottom, its intensity typically becomes much smaller than its initial intensity due to the energy absorption by the plasma plume, and hence the laser 
beam absorption and reflection at the hole bottom have been neglected. Since the focus of this paper is on the effect to the sidewall, the heat transfer from the gaseous phase to the hole bottom has been neglected. The effect of the vapor ejected from the sidewall on the gas dynamic process inside the microhole and the effect of the ejected vapor on the subsequent sidewall surface vaporization have been neglected. Also, the radiative heat transport in the plasma is not considered, and the heat flux to the sidewall only includes the heat conduction flux. It is expected that if the radiative heat flux is added, the total heat flux to the sidewall will be higher, which may increase the calculated material removal rate from the sidewall. Also, since radiative heat flux is very sensitive to the gaseous phase temperature inside the hole, it is expected that the consideration of radiative heat flux will still make the total heat flux to the sidewall non-uniform spatially, which will make the material removal from the sidewall spatially non-uniform. Hence, it is expected that if radiative heat flux is considered, it will not significantly harm the feasibility of LAPA and may even enhance its feasibility (certainly future work may still be needed to completely confirm this). The model has only calculated the material removal amount due to surface vaporization, and other possible material removal mechanisms, if exist, are expected to further increase the material removal rate from the sidewall. Also, there is certainly still room to increase the accuracy of the material properties and EOS data used in the model calculations in this paper.

\section{Conclusions}

Physics-based modeling work has been performed on the interactions among a ps laser pulse, a pre-existing plasma plume inside a microhole, and the hole sidewall. Under the 
investigated conditions, it has been found that the ps laser pulse can amplify the plasma to cause non-uniform heat flux to the sidewall and hence spatially selective material removal from the sidewall. The modeling work implies the potential feasibility of a novel dual-pulse LAPA process for drilling microholes with varying diameters at different depths. Experimental work and further modeling work are still needed to completely confirm LAPA's feasibility, fully understand its underlying physical mechanisms, and reveal its major actual advantages and disadvantages.

\section{Acknowledgement}

This material is based upon work supported by the National Science Foundation under Grant No. CMMI 1266284 and CMMI 1300930. 


\section{References}

1. Clarke, J. A., Profeta, J., "Laser micro-drilling applications", Proceedings of the 2004 Advanced Laser Applications Conference and Exposition, 2004; 2: 94-99.

2. McNally, C. A., Folkes, J., Pashby, I. R., "Laser drilling of cooling holes in aeroengines: state of the art and future challenges", Materials Science and Technology, 2004; 20: 805-813.

3. Naeem, M., "Advancement in laser drilling for aerospace gas turbines", Proceedings of the $3^{\text {rd }}$ Pacific International Conference on Application of Lasers and Optics, 2008; 197-202.

4. Wu, B., Shin, Y.C., Pakhal, H., Laurendeau, N.M., Lucht, R.P., "Modeling and experimental verification of plasmas induced by high-power nanosecond laser-aluminum interactions in air", Physical Review E, 2007; 76: 026405.

5. Gusarov, A.V., Gnedovets, A.G., Smurov, I., "Gas dynamics of laser ablation: influence of ambient atmosphere", Journal of Applied Physics, 2000; 88: 4352-4364.

6. Gurlui, S., Agop, M., Nica, P., Ziskind, M., Focsa, C., "Experimental and theoretical investigations of a laser-produced aluminum plasma", Physical Review E, 2008; 78: 026405.

7. Tannehill, J.C., Anderson, D.A., Pletcher, R.H., Computational Fluid Mechanics and Heat Transfer, 2nd ed., Washington, DC: Taylor \& Francis; 1997.

8. Tao, S., Wu, B., "Nanosecond laser pulse interactions with breakdown plasma in gas medium confined in a microhole", Applied Physics B., 2013; 113: 251-258.

9. Tao, S., Zhou, Y., Wu, B., Gao, Y., "Infrared long nanosecond laser pulse ablation of silicon: integrated two-dimensional modeling and time-resolved experimental study", Applied Surface Science, 2012; 258: 7766-7773.

10. Spitzer, L., Physics of Fully Ionized Gases, New York: Interscience Publishers, Inc.; 1956.

11. Mitchner, M., Kruger Jr, C.H., Partially Ionized Gases, New York: Wiley; 1973.

12. Harrach, R. J., "Theory for laser-induced breakdown over a vaporizing target surface", UCRL-52389, Lawrence Livermore Laboratory, Livermore, California; 1977.

13. Zel'dovich, Y. B., Raizer, Y. P., Physics of Shock Waves and High-temperature Hydrodynamic Phenomena, New York and London: Academic Press; 1966/1967.

14. Zaghloul, M.R., Bourham, M.A., Doster, J.M., "A simple formulation and solution strategy of the Saha equation for ideal and nonideal plasmas", Journal of Physics D: Applied Physics, 2000; 33: 977-984.

15. Gusarov, A.V., Smurov, I., "Thermal model of nanosecond pulsed laser ablation: analysis of energy and mass transfer", Journal of Applied Physics, 2005; 97: 014307.

16. Incropera, F.P., Dewitt, D.P., Bergman, T.L., Lavine, A.S., Fundamentals of Heat and Mass Transfer, Hoboken, NJ: Wiley; 2007.

17. Tao, S., Wu, B., Lei, S., "A comparative study of the interaction between microhole sidewall and the plasma generated by nanosecond and femtosecond laser ablation of deep microholes", Journal of Manufacturing Processes, 2012; 14: 233-242. 
18. Jeong, S.H., Greif, R., Russo, R.E., "Laser heating of metal targets including the influence of the background pressure", Proceedings of the ASME Heat Transfer Division, 1997; HTDVol. 351: 63-73.

19. Lu, Q., Mao, S.S., Mao, X., Russo, R.E., "Delayed phase explosion during high-power nanosecond laser ablation of silicon”, Applied Physics Letters, 2002; 80: 3072-3074.

20. Liu, X.D, Osher, S., "Convex ENO High Order Multi-dimensional Schemes without Field by Field Decomposition or Staggered Grids", Journal of Computational Physics, 1998; 142: 304-330.

21. McDonald, R. A., "Enthalpy, heat capacity, and heat of fusion of aluminum from $366^{\circ}$ to $1647^{\circ} \mathrm{K}$ ", Journal of Chemical \& Engineering Data, 1967; 12: 115-118.

22. Powell, R.W., Ho, C.Y., Liley, P.E., "Thermal Conductivity of Selected Materials", NSRDS-NBS 8. National Standard Reference Data Series; 1966.

23. Lemmon, E.W., McLinden, M.O., Friend, D.G., "Thermophysical Properties of Fluid Systems" in NIST Chemistry WebBook, NIST Standard Reference Database Number 69, Eds. P.J. Linstrom and W.G. Mallard, National Institute of Standards and Technology, Gaithersburg MD, 20899, http://webbook.nist.gov, (last retrieved: July 30, 2015). 


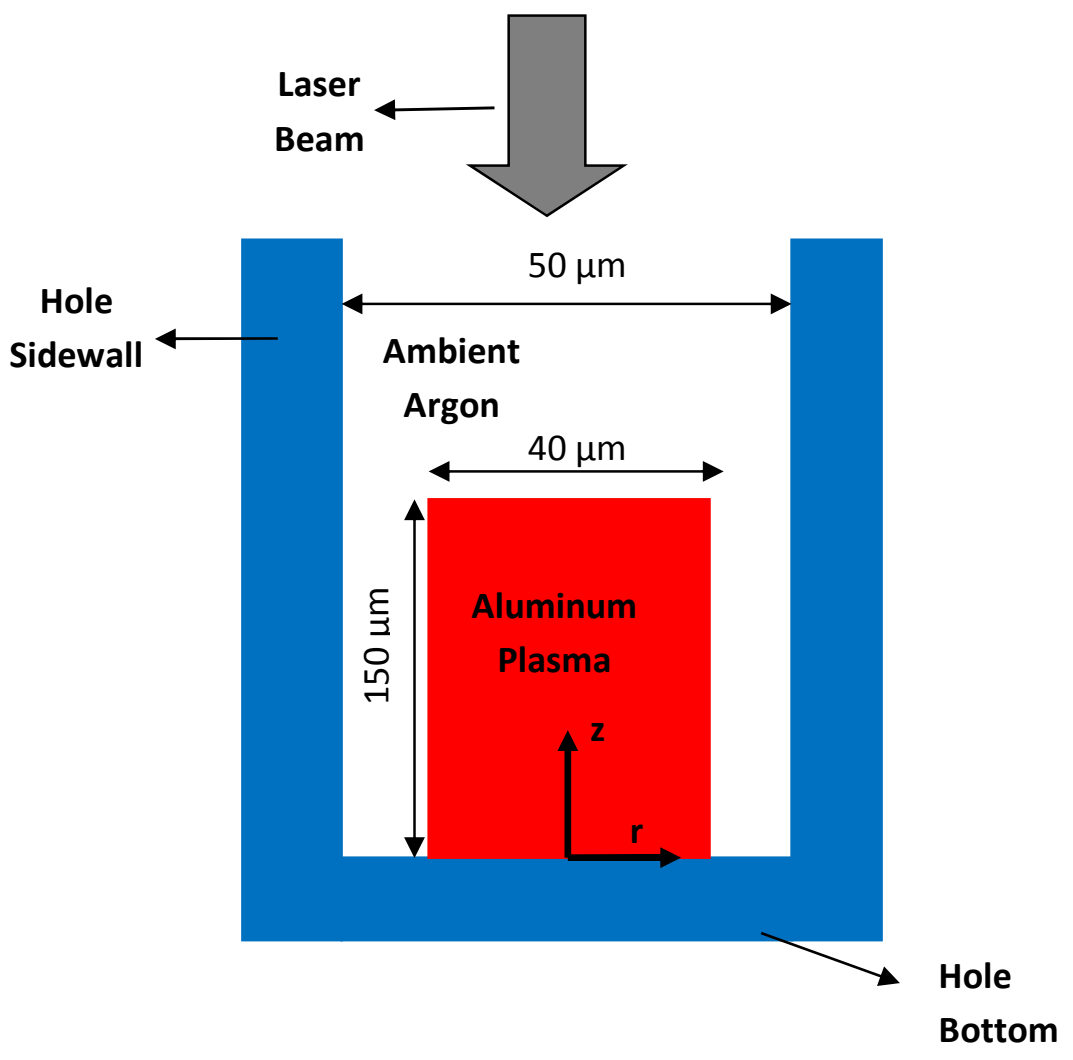

Figure 1. Schematic diagram of the model setup in this work (which also shows the initial plasma plume geometry at $\mathrm{t}=0$; the figure is not drawn to scale) 


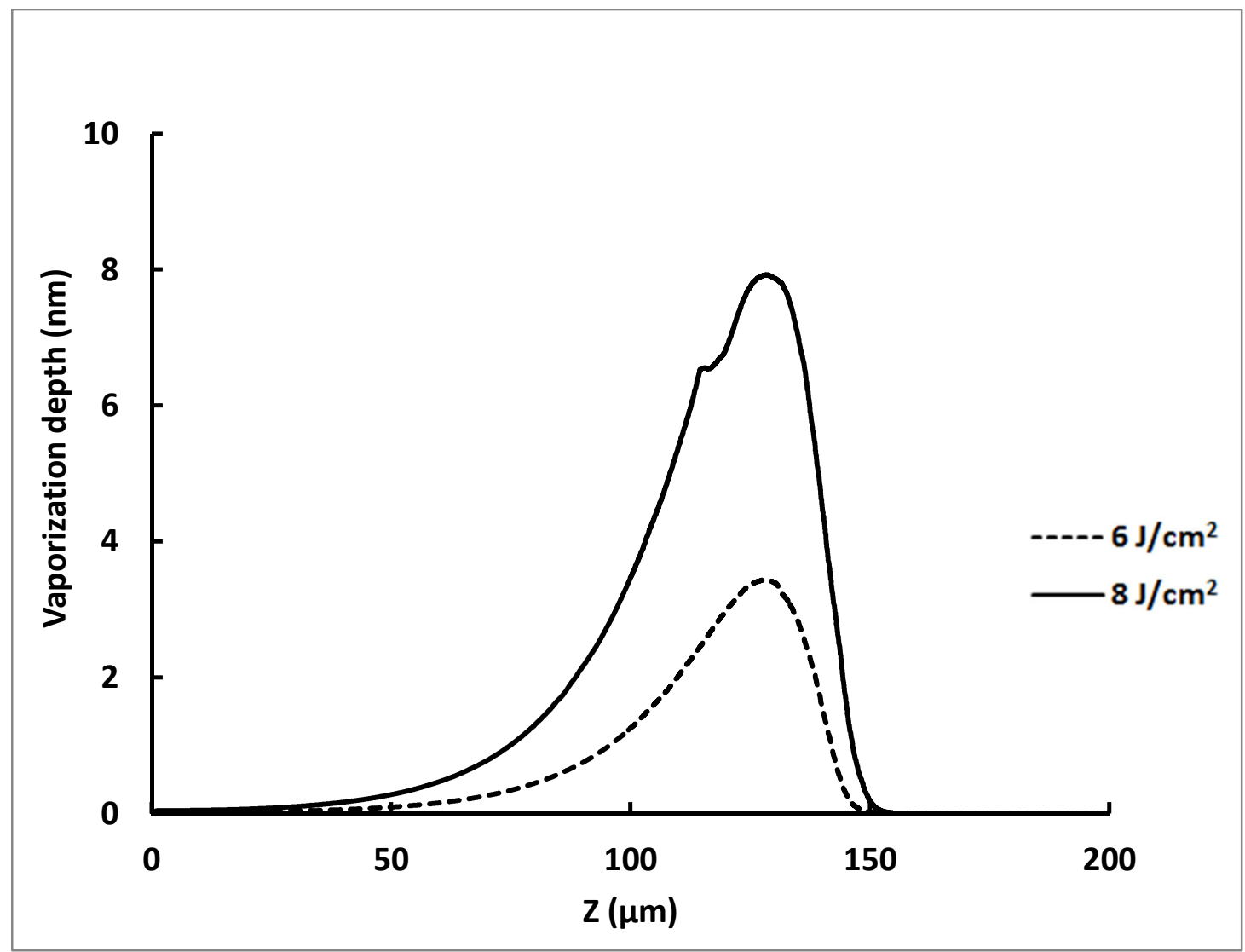

Figure 2. Spatial distribution (along $\mathrm{z}$ direction) of the model-predicted accumulated vaporization depth by $\mathrm{t}=3 \mathrm{~ns}$ for the microhole sidewall under two different picosecond (ps) laser pulse fluences $\left(6\right.$ and $\left.8 \mathrm{~J} / \mathrm{cm}^{2}\right)$. 


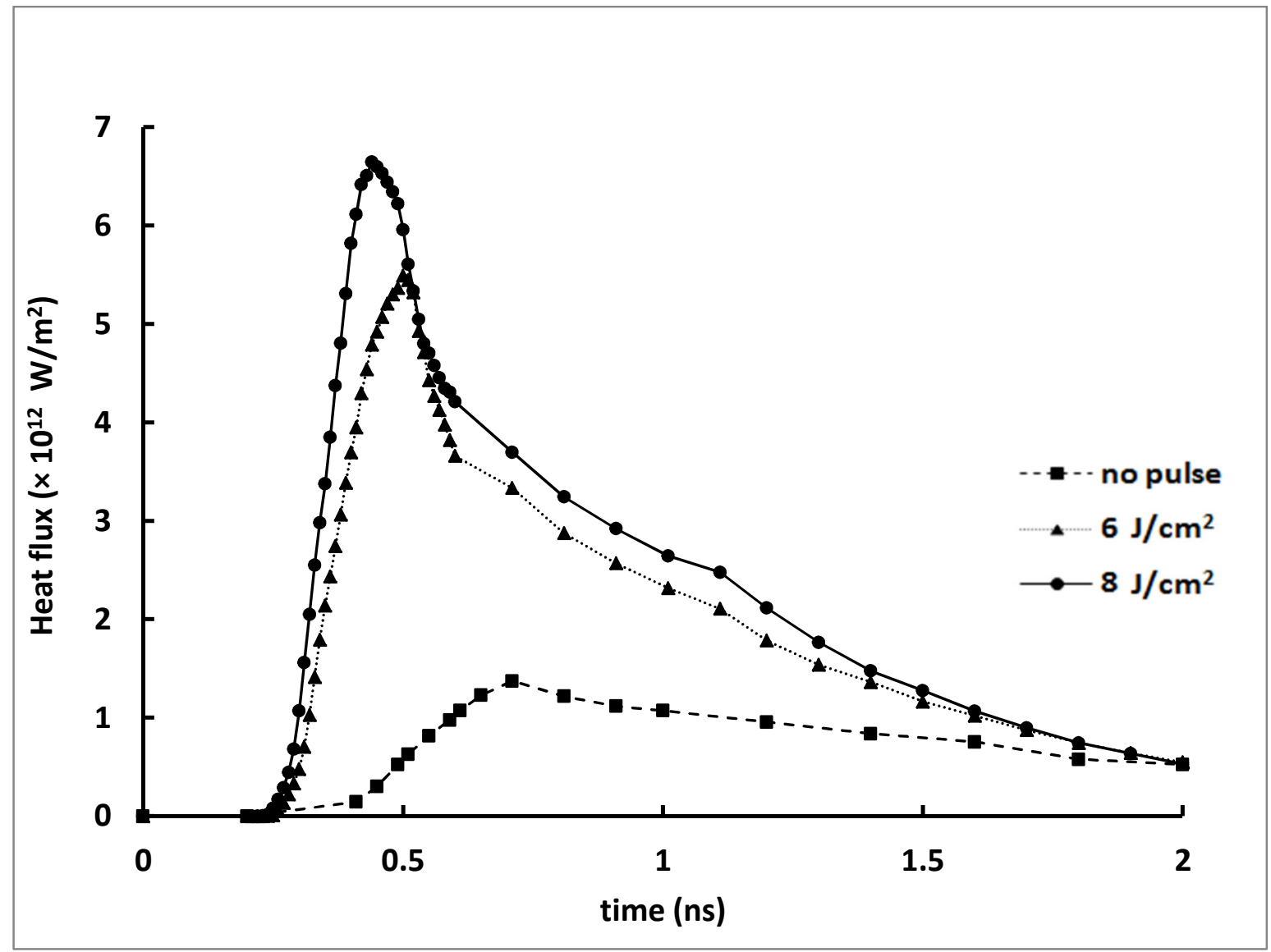

Figure 3. Model-predicted temporal evolution of the heat flux to the sidewall surface at $\mathrm{z}=128$ $\mu \mathrm{m}$, where the peak vaporization depth occurs as shown in Fig.2: (i) no ps laser pulse is applied, (ii) ps laser pulse fluence is $6 \mathrm{~J} / \mathrm{cm}^{2}$, and (iii) ps laser pulse fluence is $8 \mathrm{~J} / \mathrm{cm}^{2}$. 


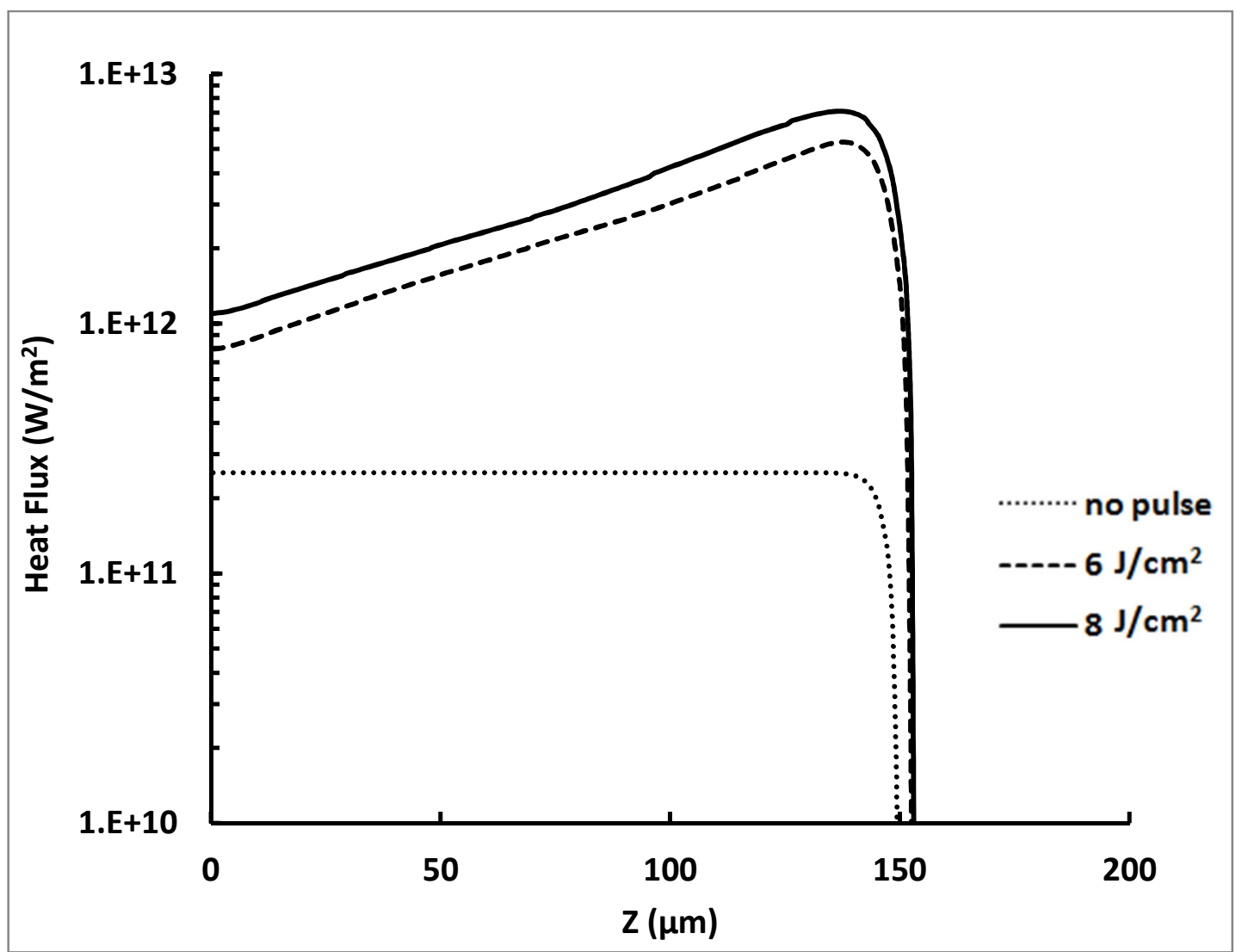

Figure 4. The spatial distribution (along $\mathrm{z}$ direction) of model-predicted heat flux to the hole sidewall surface at $\mathrm{t}=0.44$ ns: (i) no ps laser pulse is applied, (ii) ps laser pulse fluence is 6 $\mathrm{J} / \mathrm{cm}^{2}$, and (iii) $\mathrm{ps}$ laser pulse fluence is $8 \mathrm{~J} / \mathrm{cm}^{2}$. 